\title{
Adult Infectious Disease Consultation over a 10-Year Period in a Community-Base Teaching Hospital*
}

\author{
Eyassu Habte-Gabr \\ Department of Internal Medicine, Hurley Medical Center, Flint, MI, USA \\ Email: Ehabte22@yahoo.com
}

Received 28 November 2015; accepted 17 December 2015; published 21 December 2015

Copyright (C) 2015 by author and OALib.

This work is licensed under the Creative Commons Attribution International License (CC BY). http://creativecommons.org/licenses/by/4.0/

(c) (i) Open Access

\section{Abstract}

This is a descriptive review of a large number (5606) of both in and outpatient adult infectious disease consultation from a major teaching hospital over extended period (2001-2010). The purpose of the study is to get spectrum of ID and determine trends in occurrence by age and sex. The common inpatients ID consultations were SSTIs, septicemia, osteomyelitis and that for outpatient by far most common were HIV/AIDS in over a third, followed by SSTI, osteomyelitis. There was equal distribution by sex and race (African-American and Caucasian), but consultations increased with age (33\% in 60 and $32 \%$ in 45 - 59 year) especially septicemia and osteomyelitis. This data base shows spectrum of ID consultations (in and outpatient) over a long period has significance to teaching, performance for future possibly prospective studies and research priorities.

\section{Keywords}

Infectious Disease, Consultation, Hospital Admissions

Subject Areas: Infectious Diseases

\section{Introduction}

Infectious Disease (ID) remained a common cause of hospitalization and ambulatory visits in the United States [1] [2]. There has been an increasing trend of hospitalization due to ID, especially among older adults [3]-[5]). A study published in 2009 [3] showed approximately 4.5 million hospital days and $\$ 865$ billion in hospital charges associated with primary infectious disease management.

*This study was determined to be exempt from IRB review based upon 45 CFR 46.101(b) (4): Research involving the collection or study of existing data, documents, records, pathological specimens, or diagnostic specimens, if these sources are publicly available or if the information is recorded by the investigator in such a manner that subjects cannot be identified, directly or through identifiers linked to the subjects. 
Our study is a data base of inpatient and outpatient ID consultations of one infectious disease specialist's (Eyassu Habte-Gabr) experience in a major community teaching hospital. Briefly, it is a descriptive study of the overall ID burden and patterns of infection by age, gender, race and season.

All consultations were performed at patients' bedside in the hospital and in examination rooms in the ID clinic. The purpose of the consultations had been for complete management of ID of patient.

The objectives of the study a) to acquire information on spectrum of ID admissions to hospital and ambulatory visits from one ID specialist's experience; and b) analyze by age, gender and race and determine of any trends over a ten-year period.

\section{Materials and Methods}

\subsection{Study Population and Subjects}

This study was done on ID cases seen on consultation by one ID specialist over a ten year years (2001-2010) at Hurley Medical Center, Flint, Michigan. This is a 400 bed community teaching hospital and the only level I trauma center in the area. The hospital has fulltime ID specialists. Data collection was done by trained volunteers (MDs) who were given assistance and supervision by a research coordinator and the ID specialist.

\subsection{Data Source and Collection}

Information about patients was available from authors rounding cards and log books. Data collection was further acquired from inpatient and outpatients medical records and discharge summaries.

Information entered for the study included patient's age, gender, race, ID specialists consultation notes, diagnosis, predisposing factors, and comorbidities.

\subsection{Infectious Disease Classification}

IDs were classified (grouped) by site of infection namely, blood stream, skin and soft tissue, respiratory, gastrointestinal, genito-urinary cardiovascular, and neurological infections.

\subsection{Statistical Analysis}

Data analysis was done on general ID consultation trends over 10 year period by a) patient type (age , gender, and race); b) disease types by system (blood stream, respiratory etc.); and c) a separate detail analysis of ten most common infections. Results were analyzed with IBM SPSS 22.

\section{Results (Figures 1-5)}

This data base for years 2001-2010 included 5605 ID encounters. The majority were inpatient (81\%), with almost an equal distribution by gender $(\mathrm{M} / \mathrm{F}=51 \% / 49 \%)$ and race (52\% African American and 48\% Caucasian). There is a fairly good age group distribution but higher in 60 years and older (33\%), followed by 32\% in ages 45 - 59, 23.5\% in $30-44$ and $10.1 \%$ in 18 - 29 years. A small number (76 or 1.4\%) younger than 18 were also seen.

Skin and soft tissue infections were the most common cases in inpatients (27.9\%) and the second most common cases in an outpatient setting (23.3\%). A quarter of inpatients had septicemia. By far, the most common cases seen in an outpatient setting were HIV/AIDS.

Skin and soft tissue infections (27.1\%), septicemia (19.45\%), and osteomyelitis (11.98\%). Other diseases were HIV/ AIDS (9.46\%), urinary tract infection (7.63\%), respiratory tract infection (6.39\%), and febrile illness (5.48\%).

SSTIs equally affected all ages; osteomyelitis and septicemia diagnosis increased with age (Figure 3); UTI were in extreme ages (below 29 and above 60). Most HIV/AIDS patients were below 44. The major racial differences (Figure 5) are 1) SSTI higher in Caucasian (31\% vs 23\%), and 2) septicemia among African/American ( $23 \%$ vs $16 \%$ ). The main higher gender difference in ID occurrence are in 1) osteomyelitis in males (61\% vs $35 \%)$; 2) UTI in females (65\% vs $35 \%$ ) and 3) HIV/AIDS in males ( $72 \%$ vs $27 \%$ ).

\section{Discussion}

Knowledge about varieties and frequencies of ID diagnosis as seen by a specialist has significance for develop- 


\section{Most Common Infections Seen: Inpatient vs Outpatient}

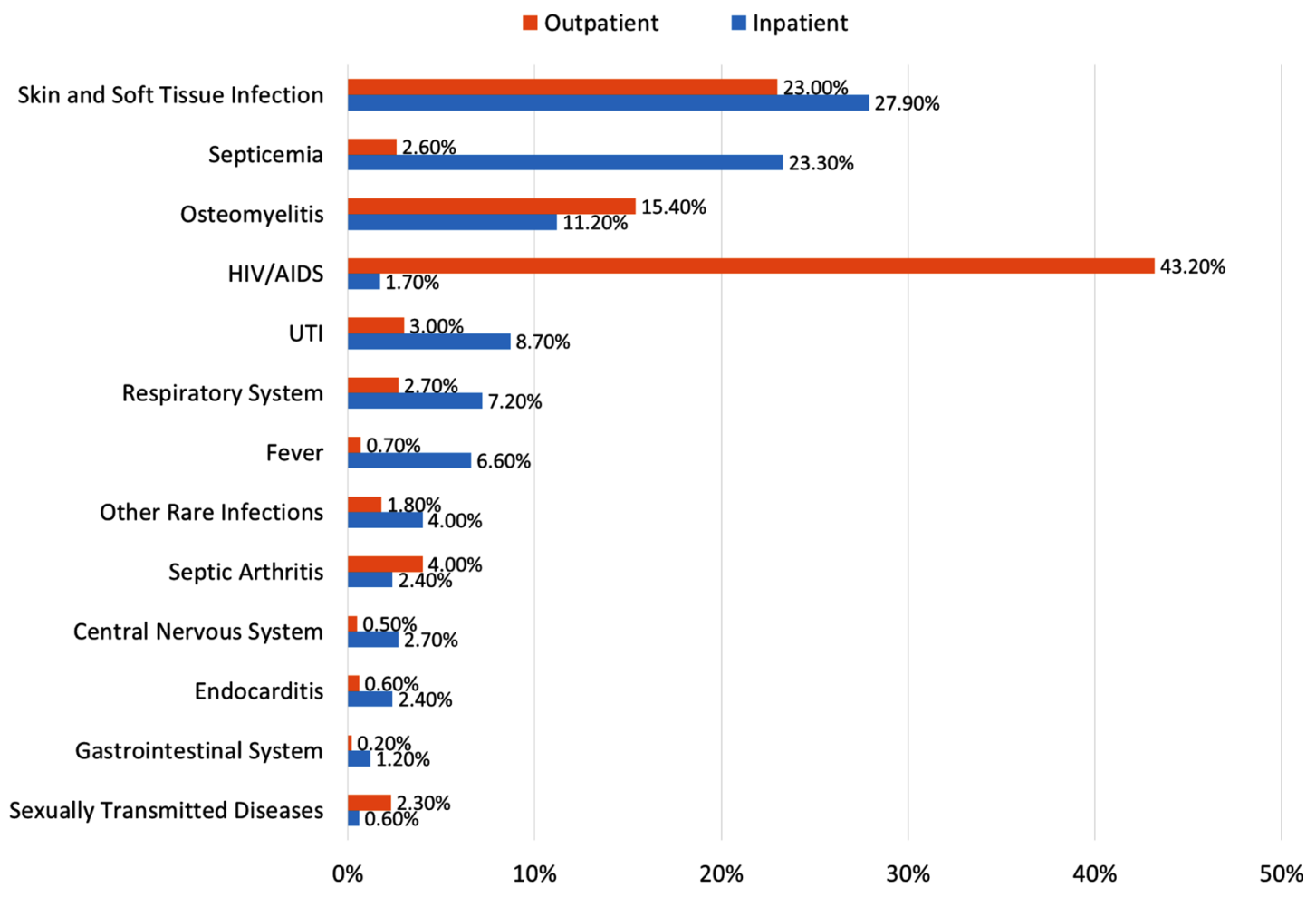

Figure 1. Disease distribution or pattern of adult ID consultation at our teaching hospital is shown.

\section{Most Common Infections Seen: Overall}

Skin and Soft Tissue Infection

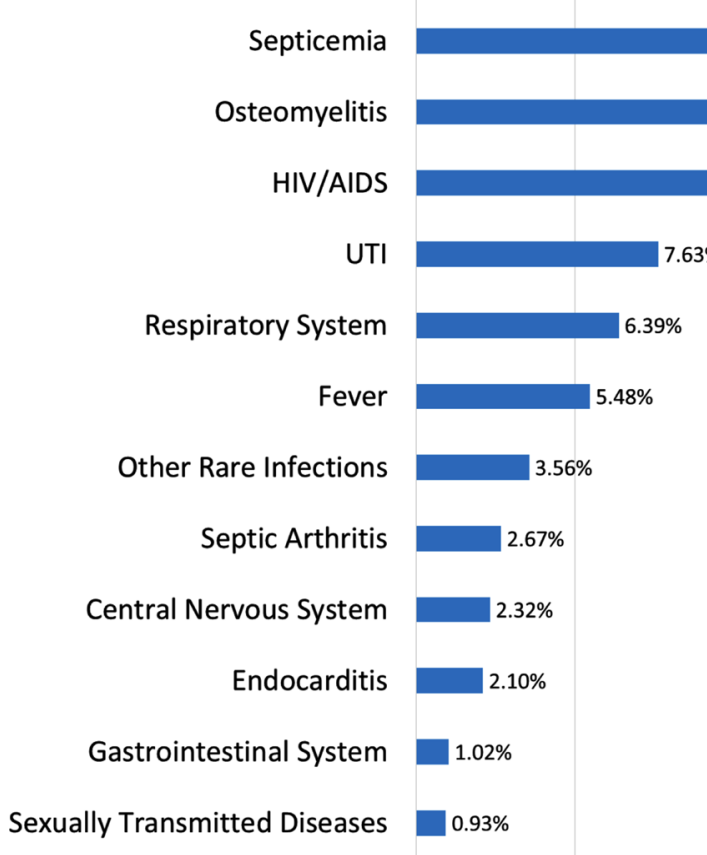

$19.45 \%$

$11.98 \%$

$$
9.46 \%
$$

$63 \%$

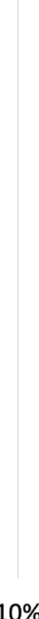

$10 \%$

$15 \%$

$20 \%$

$25 \%$

$30 \%$

Figure 2. Overall (in and outpatient) most common infections. 
Infection vs Age Group

By age group

$35 \%$

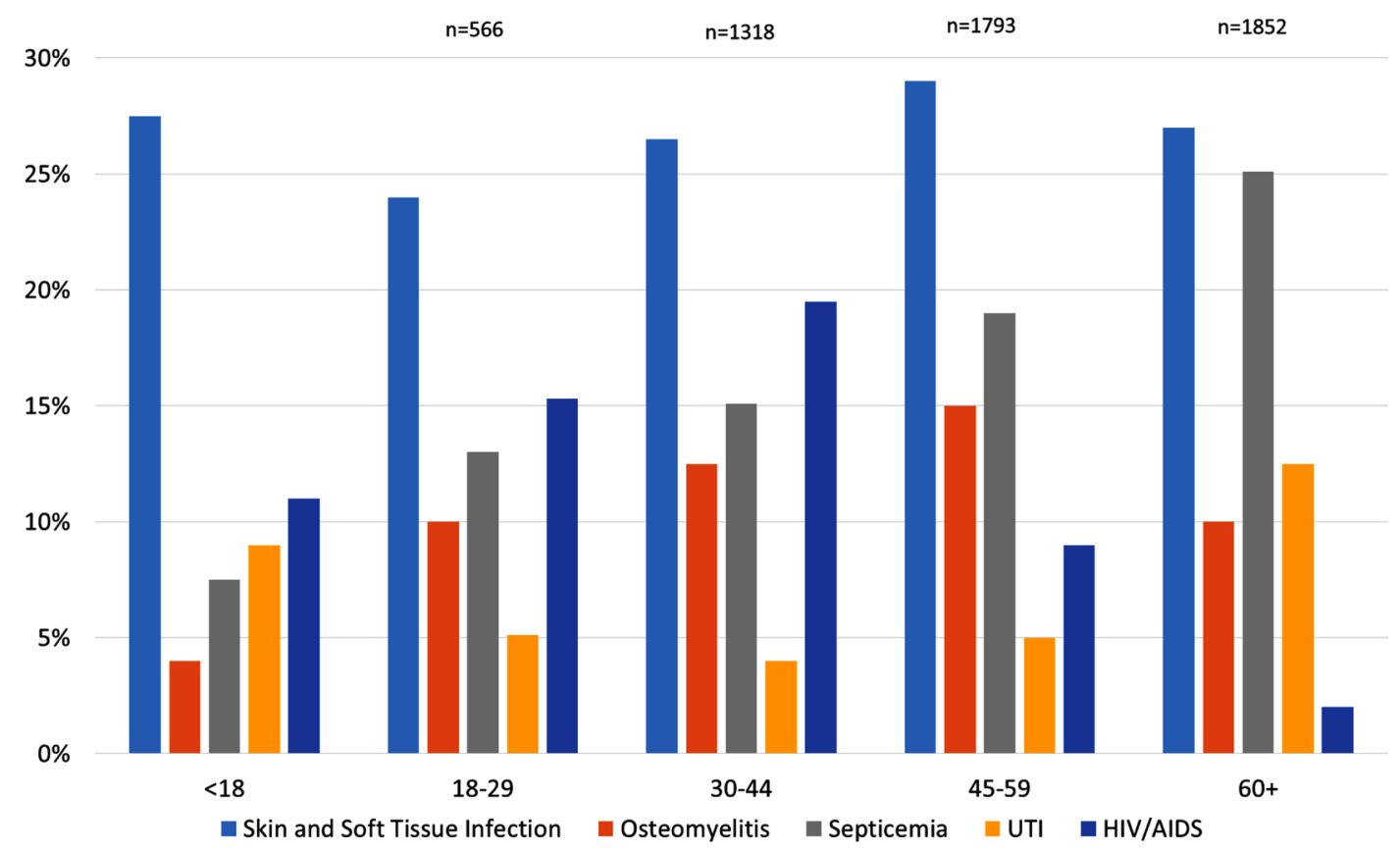

Figure 3. Distribution of ID consultation by age.

Infection vs Sex

By sex

$80 \%$

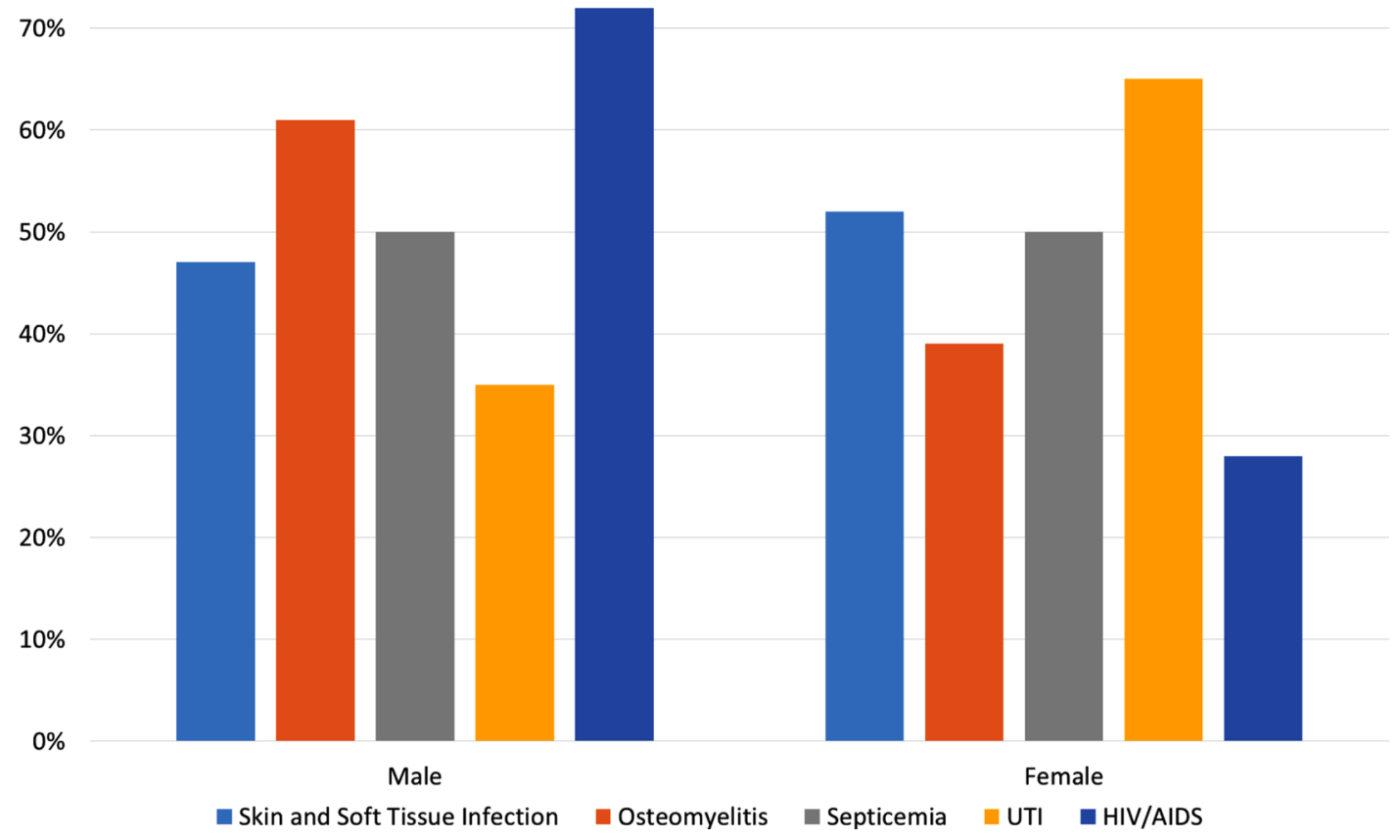

Figure 4. Distribution of ID consultation by sex. 
Infection vs Race

By race

$35 \%$

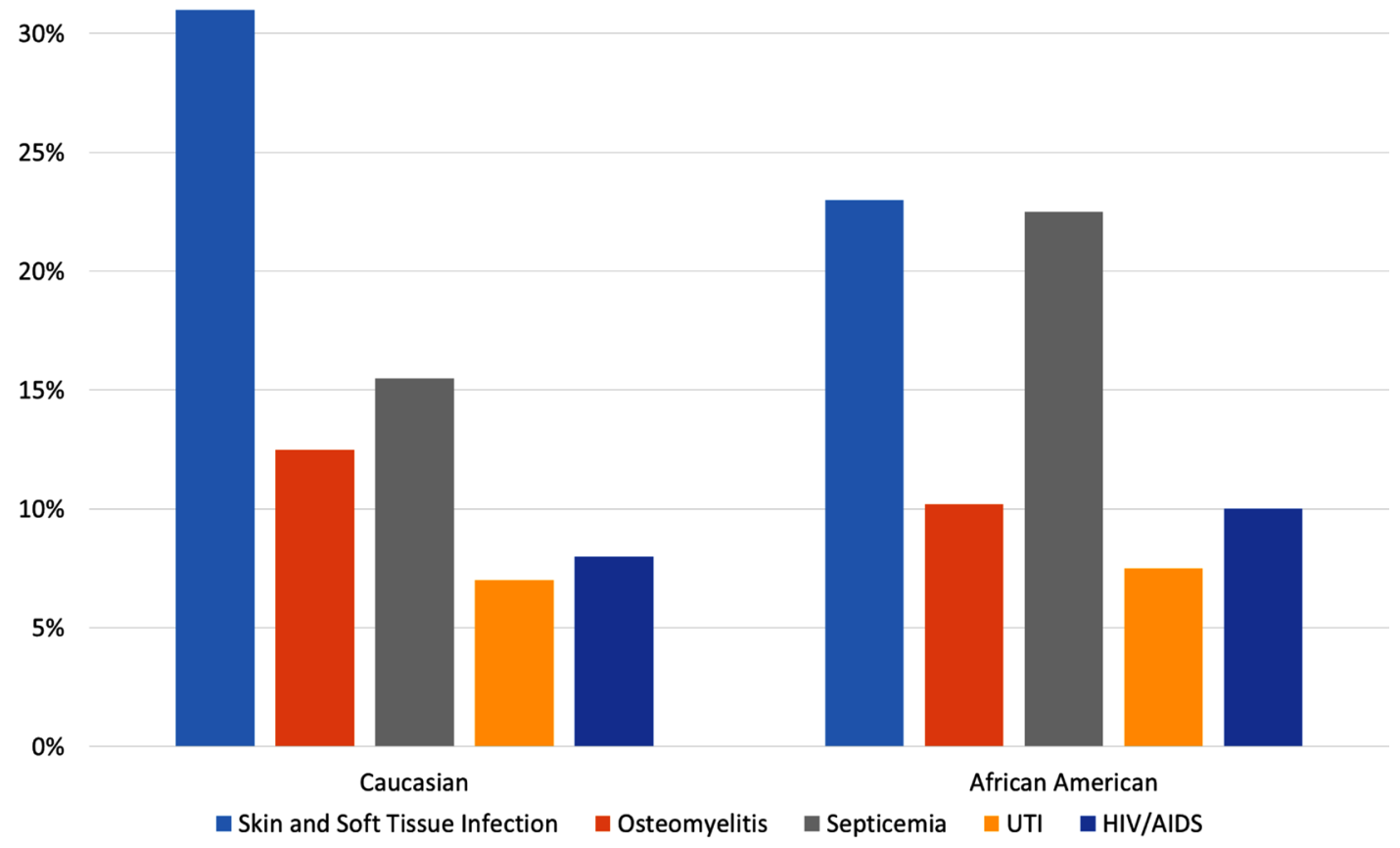

Figure 5. Distribution of ID consultation by race.

ment of teaching programs, rendering quality services, and planning for research priorities. This research on cases seen on ID consultation in a large teaching hospital, over an extended period of time, shows the reality of ID specialist daily activities in patient management and teaching of house staff and medical student in both inpatient and outpatient settings. This study is different from a study that looked at ID admissions from national data base [3], which did not specifically look at only ID consultations. For instance, respiratory tract infection ranked first but fifth in our case reviews where most patients are likely to be managed by primary care physicians and/or pulmonary specialist. Besides $20 \%$ of cases are ID consults and follow-ups at the hospital based ID clinic.

A recently published but a prospective study that we can compare our ID consultation experience was done in 53 hospitals in the Far East (Australia, New Zealand and Singapore) [6]. This was a two-week survey on ID specialists' activities. Similar to our study, skin and soft tissue infection (SSTI) was the most infection. Other common infections were respiratory tract and bone and joint infections. The shift to SSTI from respiratory tract infection has been attributed to increase prevalence of methicillin resistant Staphylococcus aureus (MRSA). There has been increasing trend in SSTI emergency department visits and admissions [7] [8].

Age and sex distribution of ID cases is as expected; women in extreme ages were more likely to have UTI and HIV/AIDS was higher in the below 44 and males. Older age population are more susceptible to infections due to decrease of T-cell function and comorbidities as seen in our hospital. There is increasing trend in the burden of ID hospitalization towards elderly in last decade [1]. This trend is clearly observed in our series more in septicemia and osteomyelitis. The increasing incidence of infections in the older age group will have a significant impact on the US health system which is expected to double by 2030.

Our study being a retrospective one has some limitations. These are unavailable/incomplete information of microbiologic data, outcome, site of acquisition of infection, details of some predisposing factors, and antibiotic use.

\section{Conclusions}

Our study reflects an experience of inpatient and outpatient consultation in a large teaching hospital during a 
regular routine year round functioning period unaffected by epidemic, population changes (example influx of returnees from abroad and unusual climatic variations. Consultations included on fairly well distributions of adult ages, race and gender. This study shows a wide spectrum of ID involving all systems.

This data collection and analysis can lead to prospective study using data base information for transfer to electronic recording of patient information, labs, imaging studies and follow-up, impact on some aspects of patient management (length of stay), cost and evaluation of antibiotic use in the hospital.

\section{Acknowledgements}

The author would like to acknowledge Jinu Puthenparampil MD for his assistance with data collection and Nicolas Lecea BS for his assistance with data analysis.

\section{References}

[1] Curns, A.T., Holman, R.C., Sejvar, J.J., Owings, M.F. and Schonberger, L.B. (2005) Infectious Disease Hospitalization among Older Adults in the United States from 1990 through 2001. Archives of Internal Medicine, 165, 2514-2520.

[2] Armstrong, C.L. and Pinner, R.W. (1999) Outpatient Visits for Infectious Disease in the United States, 1980 through 1996. Archives of Internal Medicine, 159, 2531-2536. http://dx.doi.org/10.1001/archinte.159.21.2531

[3] Yorita, K.L., Holman, R.C., Steiner, C.A., Sejvar, J.J., Stoll, B.J. and Schonberger, L.B. (2009) Infectious Disease Hospitalization in the United States. Clinical Infectious Diseases, 49, 1025-1035.

[4] Simonsen, L., Conn, L.A., Pinner, R.W. and Toutsch, S. (1998) Trends in Infectious Disease Hospitalizations in the United States, 1980-1994. Archives of Internal Medicine, 158, 1923-1928.

[5] Saliba, W., Fedial, A., Edelstein, H., Markel, A. and Raz, R. (2013) Trends in the Burden of Infectious Disease Hospitalization among the Elderly in the Last Decade. European Journal of Internal Medicine, 24, 536-540. http://dx.doi.org/10.1016/j.ejim.2013.06.002

[6] Ingram, P.R., Cheng, A.G., Murray, R.J., Blyth, C.C., Walls, T., Fisher, D.A. and Davis, J.S. (2014) On Behalf of the Australian Society for Infectious Disease Clinical Research Network. What Do Infectious Diseases Physicians Do? A 2-Week Snapshot of Inpatient Consultative Activities across Australia, New Zealand and Singapore. Clinical Microbiology and Infection, 58, 1-8.

[7] Hersh, A.L., Chambers, H.E., Masel, J.H. and Gonzales, R. (2008) National Trends in Ambulatory Visits and Antibiotic Prescribing for Skin and Soft Tissue Infections. Archives of Internal Medicine, 168, 1585-1591. http://dx.doi.org/10.1001/archinte.168.14.1585

[8] Pallen, D.J., Egan, D.J., Pelletier, A.J., Espinola, J.A., Hooper, D.C. and Camargo Jr., C.A. (2008) Increased US Emergency Department Visits for Skin and Soft Tissue Infections and Changes in Antibiotic Choices, during the Emergency of Community-Associated Methicillin-Resistant Staphylococcus aureus. Annals of Emergency Medicine, 5, 291-298. 